\title{
Tackling immobility in hospitalized seniors
}

A

nyone who has walked through a hospital ward has seen room after room of elderly patients quietly dozing in bed, as if every patient was being treated for a cold or hysteria, or recumbence the necessary condition of all therapies.

And doesn't it make sense, after all, that elderly patients should spend their days on their back? Traditional wisdom dictates that bedrest promotes healing and recovery, much in the manner that "rest in bed and drink plenty of fluids" is the solution to the common cold and an extended bout under the covers was the definitive answer in the 19th century to a diagnosis of hysteria.

But it may be time to put the bedrest prescription to bed and get those seniors moving through the wards, as studies indicate that elderly, hospitalized patients are more likely to be discharged to long-term care facilities, or even to die, as a result of the deleterious effects of prolonged bedrest, including "hospitalizationassociated disability," i.e., the loss of ability to complete one or more of the basic activities needed to live independently, such as bathing, dressing and using the toilet.

"Patients often leave the hospital worse than they came in. It really raises questions to me about what we're doing in the hospital environment," says Dr. Cynthia Brown, a geriatrician at the University of Alabama at Birmingham and a researcher at the Birmingham/Atlanta Veterans Affairs Geriatric Research, Education and Clinical Center.

"We know that bed rest in and of itself is associated with numerous adverse outcomes, many of which we are trying to prevent, like pressure ulcers, deep vein thrombosis and functional decline," adds Brown, whose studies indicate that an elderly patient typically spends a staggering $83 \%$ of the time he is hospitalized lying on his back. Brown and colleagues attached wireless accelerometers to hospitalized seniors and found that they spent just

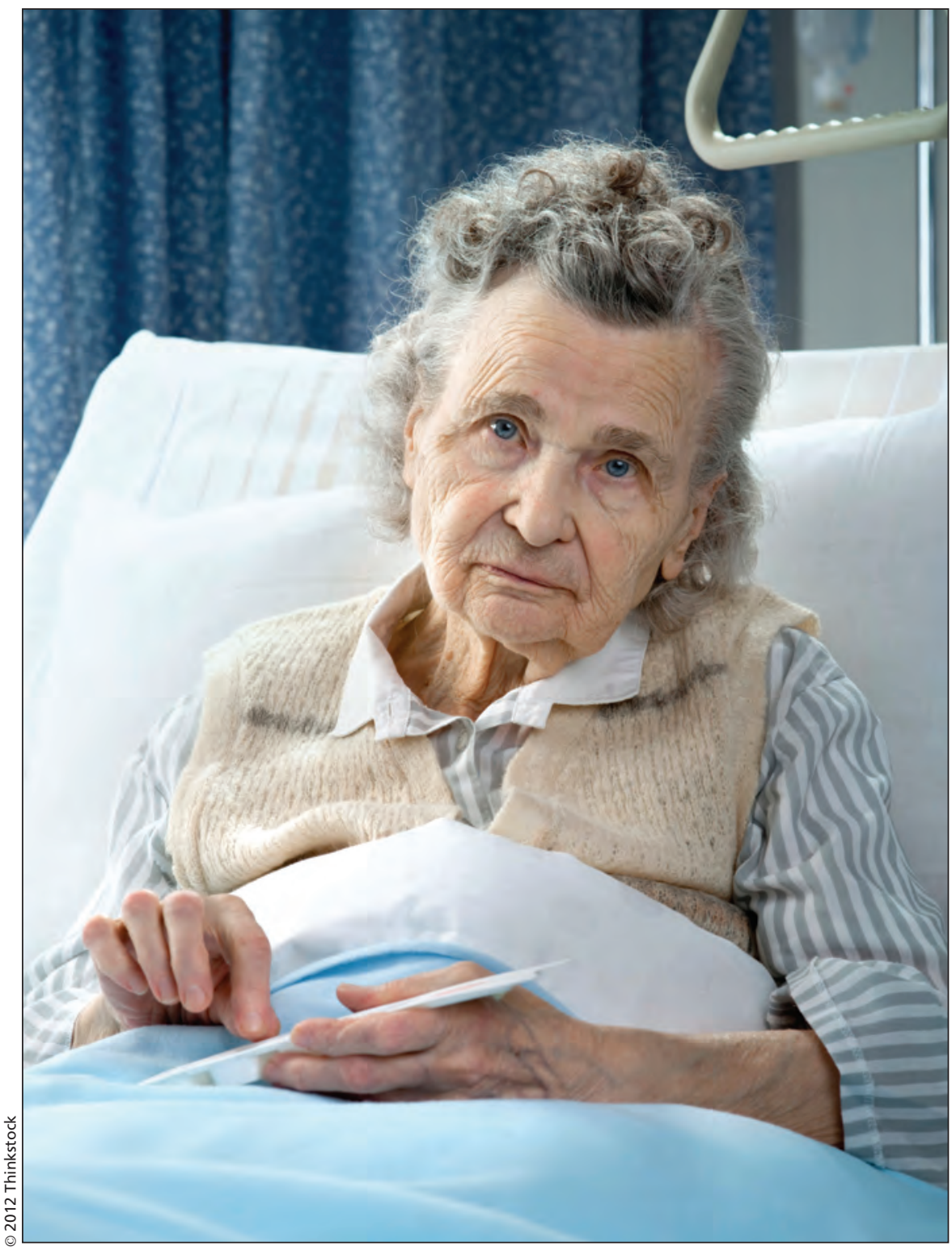

"Patients often leave the hospital worse than they came in. It really raises questions to me about what we're doing in the hospital environment," says Dr. Cynthia Brown, a geriatrician at the University of Alabama at Birmingham.

$3 \%$ of their time standing or walking $(J$ Am Geriatr Soc 2009;57:1660-5).

Indeed, the evidence has long been compelling that prolonged bedrest for a senior is hazardous. Hospitalized seniors lose up to $5 \%$ of their muscle strength daily (Ann Intern Med 1993;118:21923). Compared with more active and mobile hospitalized counterparts, immobile seniors are six times more likely to be discharged to long-term care facilities and 34.3 times more likely to die in hospital ( $J$ Am Geriatr Soc 2004;52:126370). In one study, some 799 of 2279 , or $35 \%$, of hospitalized elderly patients were discharged with hospitalizationassociated disability and within a year, $41.3 \%$ of those had died and only $30.1 \%$ were at baseline function ( $\mathrm{J}$ Am Geriatr Soc 2008;12:2171-9). 
The short answer is to get those seniors moving, as early as possible while in hospital, Brown says. "We need to be jumping on this mobility issue from the moment they come in the door and not waiting until discharge and realizing that they're not capable of walking anymore."

But hospitals typically aren't designed, or inclined, to promote mobility, she notes. "The television is directly over the bed. Often times, there's only one chair in the room, and so if you have company you're going to stay in the bed, and people aren't really encouraged to sit up for meals because we just don't think about it."

There's also a lack of destinations of interest to patients, Brown notes. "We've talked about having some sort of destination in the VA [Veteran Affairs System], for example, a big screen TV playing the football game, some place that is more interesting to walk to."

Brown notes that several newer units for acute care of the elderly are being designed with communal dining areas, to ensure that patients get moving at least three times a day.

Another solution that Brown's facility has adopted is appointing a cohort of "walking aides" whose mission is to persuade patients to move. "If you get walking aides who are entertaining, then it becomes a social event. There are ways to make it interesting. If we walk in silence that's boring, if we walk and talk then it becomes much more acceptable."

Several facilities in Ontario are also exploring means of improving senior mobility as part of a decision in September 2011 to make the promotion of early mobilization as a clinical priority of the Senior Friendly Hospital Strategy of the province's Local Health Integration Networks.

"The goals of the intervention are to ensure that people are assessed early, within 24 hours of admission, and to encourage mobility to the maximum potential of the patient three times a day," says Dr. Barbara Liu, executive director of the Regional Geriatric Program of Toronto and co-leader of MOVE ON (Mobilization of Vulnerable Elders in Ontario).

The MOVE ON initiative has developed a series of educational modules, posters, worksheets, assessment algorithms and patient handouts that have been made available in 14 academic hospitals and are adaptable to "different hospital units and different patient populations," Liu says.

At Sunnybrook Health Sciences Centre, where Liu works as a geriatrician, the facility has developed a volun- teer program to promote mobility among the elderly and redesigned the environment to promote mobility. "We created a checklist for reducing clutter in the hallways" and making it easier for patients to safely navigate hallways by using handrails, Liu says. "On some of our floors we have these chair stations that are situated around the unit. These are places where people can sit down and rest. And these chair stations also have some suggested exercises that you can perform while sitting."

Staff and patients appear to have responded enthusiastically. One patient, a 65-year-old woman named Linda, who asks that her surname not be disclosed, credits the MOVE ON program with preventing her functional decline after being hospitalized as a result of fall. Initially confined to a wheelchair, she found herself moving with the assistance of a walker after two weeks, and her wheelchair consigned to a corner. "I feel a lot more mobile, a lot more secure," she says. "You gotta walk, if you don't walk you're in big trouble. Just generally speaking, it makes sense. We've walked all our lives. It's no good to anybody young or old to not walk." - Nathan Stall MD, Toronto, Ont.

CMAJ 2012. DOI:10.1503/cmaj.109-428

\section{National standards urged for end-of-life care providers and facilities}

$\mathrm{P}$ op quiz. If someone wants to start administering palliative, end-of-life or home care in Canada to the terminally ill or elderly, do they need training? Certification? Accreditation? Are they obliged to periodically demonstrate competence or compliance with any manner of standards?

The short answer to all of the above?

For the most part: No.

"Anybody can hang out a shingle and say they're palliative care and nobody can quibble with them," says Dr. Larry Librach, Sun Life Financial
Chair in Bioethics at the University of Toronto in Ontario and past director of the Temmy Latner Centre for Palliative Care at Mount Sinai Hospital.

The absence of national standards and legal requirements essentially means that, in most parts of the country, almost anybody can set themselves up as a provider of the nonpharmaceutical facets of end-of-life care, on an almost fly-by-night basis, even if they lack the resources or training to manage the myriad complexities associated with providing care.

But while Canada isn't exactly overrun with erstwhile providers, sporadic reports of problems and abuses associated with end-of-life and home care indicate that the introduction of standards would elevate the quality of the industry, experts say.

Accreditation of health care providers and facilities is mostly voluntary in Canada. There are few exceptions. It is required in all public and private health care institutions in Quebec, and all facilities supported by regional health authorities in Alberta. Manitoba is also in the process of developing legislation that would compel providers and facilities within regional health authorities to obtain and maintain accreditation. 